\title{
REVIEWERS OF ASHS MANUSCRIPTS, 1994
}

Every paper in the Journal of the American Society for Horticultural Science is reviewed by at least two scientifically qualified persons ASHS is pleased to publish the following list of members and other individuals who reviewed manuscripts for the 1994 issues. Tht Society appreciates the time and unselfish effort rendered by reviewers and acknowledges their contribution in making ASHS publications scientifically reliable and useful.

Judith A. Abbott

Aref Abdul-Baki

William B. Ackley

Carol J. Adams

Stuart W. Akers

Mary Lewnes Albrecht

Peter C. Andersen

Robert L. Andersen

Robert G. Anderson

Douglas Archbold

P.G. Arnison

Michael A. Arnold

Mary Lu Arpaia

Edward N. Ashworth

L.R. Askham

Robert M. Augé

James R. Ault

J. Bacher

Sayed A. Badr

J.R. Baggett

Douglas A. Bailey

J.T. Baker

James R. Ballington

T.J. Banko

Nigel H. Banks

D.J. Barbara

Cynthia L. Barden

John A. Barden

Allen Barker

Reed E. Barker

Bruce Barrett

James E. Barrett

Richard Barret

Daniel Barta

Mark J. Bassett

Ernst Baumann

David J. Beattie

R. Beaudry

James Beaver

Tom Beckman

Richard Bell

Robin Bellinder

David Bender

Mark A. Bennett

Brian Benson

Mark Beritt

Wade Berry

Isa Bertling

Richard Bessin

Caula A. Beyl

Prasanta C. Bhowmik

John A. Biernbaum

Ted Bilderback

Thomas Bjorkman

Alfred Blackmer

Frank A. Blazich
Frederick A. Bliss

Paul Bosland

John Bouwkamp

Dan Bowman

Kim D. Bowman

Charles Boyer

John J. Bozzola

Kent J. Bradford

William J. Bramlage

Mark H. Brand

S.E. Branen

Jeffrey K. Brecht

James L. Brewbaker

Mark Bridgen

Timothy Broschat

C.R. Brown

G.R. Brown

G.S. Brown

Patrick Brown

Susan Brown

Robin Brumfield

Blair Buckley

Bruce Bugbee

David Burgard

David W. Burger

D.M. Burgett

D.H. Byrne

Gustavo Caetona-Anolles

Charles R. Caldwell

Craig C. Camaday

Scott Cameron

Craig A. Campbell

Richard J. Campbell

Carl Cantaluppi

William H. Carlson

William J. Carpenter

Mark Carroll

N.B. Carroll

Anne Carter

Bob Cartwright

Catherine G. Cavaletto

R.F. Cerkauskas

Richard B. Chalfant

Oyette L. Chambliss

Harvey Chan

Craig K. Chandler

Paula P. Chee

Paul Chen

Tony H.H. Chen

Zongming Chen

Nick Christians

Dina St. Clair

John Clark

Greg Cobb

David Coffey

Charles W. Coggins
Philip F. Colbough

Maria T. Colinas-Leon

Wanda W. Collins

Robert R. Coltman

Michael Compton

William S. Conway

Tom Cook

K.A. Corey

Brian E. Corr

Donald J. Cotter

Melvin Couey

Douglas A. Cox

Dermot P. Coyne

Richard Craig

Jonathan Crane

Richard A. Criley

Carlos Crisosto

Thomas Crocker

Michelle Crouse

George A. Cummings

John Cummings

Eric A. Curry

Steven Czaplewski

Frank J. Dainello

Adam Dale

Hugh A. Daubeny

Margorie Daughtrey

Thomas Davenport

Frederick S. Davies

F.T. Davies, Jr.

David W. Davis

Jeanine M. Davis

Tim D. Davis

Kevin R. Day

Bill D. Dean

Dennis Decoteau

August A. DeHertogh

Ted DeJong

M.J. Delwiche

Carolyn DeMoranville

Frank G. Dennis, Jr.

Peter H. Dernoeden

Dale A. Devitt

Robert M. Devlin

Murli Dharamadhikari

J. Dick

Michael H. Dickson

Michael A. Dirr

Michael Dixon

Nick Dokoozlian

John M. Dole

Paul A. Domoto

Henry Douselman

C.G. Downs

Stephen R. Drake

Dan Drost
A.E. Dudeck

Robert J. Dufault

Richard E. Durham

Dominic Durkin

Edward F. Durner

James Duthie

Elisabeth Earle

George Eaton

Mark Ehlenfeldt

Mohamed El-Otmani

George Elliot

Monica L. Elliott

Dave Ellis

Clyde E. Elmore

Gary Elmstrom

Mary Jane Else

Charlie Embree

George C. Emery

Michael Evans

Richard Evans

Robert G. Evans

Kathleen B. Evensen

James Everitt

Elmer Ewing

Miklos Faust

Peter Feretti

G.C.J. Fernandez

Tom Fernandez

David Ferree

Peter A. Ferretti

Richard Fery

C. Fideghelli

Debbie M. Filler

W.E. Finch-Savage

Chad E. Finn

Keith J. Fisher

Jack B. Fisher

Harrson Flint

James A. Flore

William C. Fonteno

Jerome L. Frecan

Cal A. Froberg

Jack D. Fry

Leslie H. Fuchigama

Patrick Fuerst

K.A. Funnell

Richard C. Funt

Sheldon Furutani

Lena Gallitano

Melvin P. Garber

J. Thomas Garrett

Stephen A. Garrison

Stephen Garton

Karen L.B. Gast

Paul Gepts

Dianne Gerlach 
T.L. German

Hans Gerritsen

David A. Gilbertz

K.L. Giles

Ricahrd J. Gladon

Fred G. Gmitter

Joe Gofreda

I. Goldman

Alfred Gonzales

André Gosselin

Robert Gough

Francis R. Gouin

Barbara L. Goulart

Thomas Gradziel

Steve Grattan

Dennis Gray

Duane W. Greene

Robert J. Griesbach

M.G.J.M. van Grinsven

Earl Gritton

David Groff

Jude Grosser

Yaffa Grossman

Rebecca Grumet

P.K. Gupta

R.P. Guries

Wesley P. Hackett

Dave Hahn

John Halbrendt

Charles Hall

P. Allen Hammer

Freddi Hammerschlag

Susan S. Han

J. F. Hancock

Lee Handley

Brent Harbaugh

Joan P. Harris

Helen Harrison

T.K. Hartz

Jonathan Haskett

Michael J. Havey

Kathleen Haynes

W.W. Heck

Royal D. Heins

Robert Heisey

Jimmy Hendrix

Richard J. Henny

Paul H. Henry

David Hensley

Robert C. Herner

David Hershey

John D. Heskett

Peter Hicklenton

James R. Hicks

David F. Hildebrand

David G. Himelrick

Roger Hinson

George J. Hochmuth

Laurie Hodges

E.J. Holcomb

Roger Hoopingardner

Emily Hoover

Teresa K. Howe
Charles Howell

Greg Hoyt

Mark Hubbard

David R. Huff

Harrison G. Hughes

Jerome Hull, Jr.

Rita Hummel

David Hunter

Amy F. Iezzoni

Jeffrey Iles

Russell E. Ingham

Morris Ingle

Betty Ishida

Shuichi Iwahori

Larry K. Jackson

Andrew R. Jamieson

Harry Janes

Jules Janick

Robert L. Jarret

Richard Jaynes

B.J. Johnson

Johnny Johnson

Jon R. Johnson

Scott Johnson

R. Jolly

Robert Joly

Alfred Jones

Eugene Jones

Harry Jones

E.L. Jourdain

Pablo S. Jourdan

Gary Judd

Mark P. Kaczperski

Adel Kader

Brian Kahn

Tracy Kahn

Walter Kaiser

Willy Kalt

Michael E. Kane

Martin Kaps

Robert F. Kasmire

Stanley J. Kays

D. $\mathrm{Ke}$

Dang Yang Ke

Ed Kee

Gary J. Keever

James H. Keithley

Keith Kelling

Anwar A. Khan

Michael Kilby

Larry D. King

Ann King

S.L. Kitto

J.D. Klein

James E. Klett

W. Mark Kliewer

Gary Kling

Steve Knapp

Michael Knee

Larry Knerr

Robert J. Knight

Moritz C. Knoche

John Koa
S.S. Korban

John M. Kraft

Don Krizek

Robert Kruger

Lorin R. Krusberg

Mosbah Kushad

Molly Kyle

Alan N. Lakso

Michel Lamarre

Elizabeth Lamb

Mary L. Lamberts

Calvin Lamborn

James A. LaMondia

William J. Lamont, Jr.

Benoit Landry

Ron Lane

Robert Langhans

M. Larean

Fenton Larson

Roy Larson

Daniel Laskovar

Joyce G. Latimer

O.L. Lau

Michael Lay-Yee

Desmond R. Layne

R.E.C. Layne

Chinon Lee

Daniel Leskovar

Charles Leslie

Heinrich Lieth

David Lightfoot

R.E. Lill

Orville M. Lindstrom

Norman E. Looney

Richard Lower

Brent Loy

James Luby

S. Lurie

Paul Lyrene

Eugene V. Maas

Bradley A. Majek

Joseph J. Makhlouf

Don Makus

Michael Marcotrigiano

Richard P. Marini

Lurline Marsh

George C. Martin

MasaruTsukita

John Masiunas

Helena Mathews

Bernie May

Roger May

Brian K. Maynard

Donald N. Maynard

Lambert B. McCarty

Charles A. McClurg

Gregory McCollum

Robin McConchie

Patrick McCool

Brent H. McCown

Deborah McCown

James D. McCreight

Roy E. McDonald
M.B. McDonald

Jim McFerson

Barry McGlason

Margaret McGrath

B.D. McGraw

Bruce D. McKersie

Mark McMurtry

Alan W. Meerow

James Menzies

F. Meredith

Scott A. Merkle

Richard H. Merritt

Warren C. Micke

E. Mielke

Phillip N. Miklas

N. Miles

Dianne D. Miller

J.C. Miller

Raymond A. Miller

William B. Miller

Harry Mills

Gaylord Mink

Peter Minotti

Elizabeth Mitcham

Cary A. Mitchell

Gordon Mitchell

Teresa Mitzel

David Mok

Gloria Moore

James N. Moore

Patrick P. Moore

Renae Moran

Teddy E. Morelock

J.R. Morris

Robert C. Morrow

Desmond G. Mortley

James E. Motes

Fred Muehlbauer

Charles Mullins

Norimoto Murai

Tim Murphy

James Myers

Russell T. Nagata

Henry J. Nakasone

Terril Nell

Hsim Nerson

D.S. NeSmith

Steven Earl Newman

R.J. Newton

Courtland Nichols

A.X. Niemiera

Jeffrey G. Norcini

Jeffery Norcini

Perry Nugent

Richard O'Barr

Charles O'Dell

Norman Oebker

Alex Ogg

W. Okie

William C. Olien

William J. Olien

Stephen Olson

Douglas Ormrod 
Michael Orzolek

Allen D. Owing

John Pair

Noel E. Pallais

Michael Parker

Kim D. Patten

Mary M. Peet

Ellen Peffley

Harold Pellett

Norman E. Pellett

Brent Pemberton

Leonard Perry

Ron Perry

Peter Petracek

Paul L. Pfahler

Sharad C. Phatak

H. Wayne Philley

R.D. Phillips

David Picha

Dennis Pittinger

F. Pokorny

Vito Polito

Barcley Polling

Robert Pool

Hugh A. Poole

Wayne C. Porter

John Potter

Lloyd Powell

Vaino W. Poysa

Hugh Price

Marvin P. Pritts

Jean-Pierre Privé

Edward L. Proebsting

Rosario Provvidenti

Stan Prussia

Dan Purcifull

Albert Purvis

H.A. Quamme

Carlos Quiros

Jonathan R. Schultheis

David Ramming

David E. Ramos

Don Ramsdell

William Randle

Thomas Rathier

Dennis Ray

Paul E. Read

Barbara M. Reed

Michael Reid

W. Reid

Stephen Reiners

Bruce Reisch

Billy B. Rhodes

Charles M. Rick

Mark Rieger

Paul E. Rieke

Kathy Rietsma

David F. Ritchie

Carol D. Robacker

Jo Ann Robbins

Warren Roberts

John Robinson

R.W. Robinson
Terence L. Robinson

Mark Roh

Curt R. Rom

C. Peter Romaine

Mikeal L Roose

Teryl Roper

Carl Rosen

Philip Rowe

Lisa Rowland

Vincent Rubatzky

John M. Ruter

Glen Ruttencutter

Edward J. Ryder

John Sacalis

Roy M. Sachs

Yoneo Sagawa

John C. Sager

Mikal Saltveit

Carl Sams

Charles Sanchez

Douglas Sanders

Kenneth C. Sanderson

Joseph C. Scheerens

Michael Schnelle

Mary Schon

Steven H. Schroeder

Ursula Schuch

Jonathan Schultheis

Jeffrey Scott

John W. Scott

Simon Scott

William J. Segars

Andrew Senesac

Ken Shackel

Carl Shanks

Douglas Shaw

Thomas J. Sheehan

Krista Shellie

Dennis Shepard

Wayne Sherman

Philipp Simon

Kenneth Sink

Arie Sive

Thomas M. Sjulin

Robert M. Skirvin

R.W. Smiley

Michael Smith

R.B. Smith

Rex L. Smith

George H. Snyder

Theo Solomos

Darrell Sparks

Sara Spayd

James Spiers

W.E. Splitstoesser

David M. Spooner

Thomas Sporleder

Tery Starman

James Steadman

Susan Steinberg

Dow Steinegger

Alan Stemler

Susan B. Sterrett
Herb Stiles

Steve Still

Dennis Stimart

Peter J. Stoffella

John R. Stommel

J. Stowe

John Strang

B. Strik

Daniel Struve

Cecil Stushnoff

J. Alan Sullivan

Ellen G. Sutter

B.T. Swanson

Dariusz Swietlik

S.R. Sykes

Fumiomi Takeda

Mei-Lie Tam

Michael J. Tanabe

S.M. Tavantzia

Alan G. Taylor

Glenn G. Taylor

John Teasdale

Paul A. Thomas

Paul G. Thompson

M. Thornton

M.K. Thornton

T.W. Tibbitts

P. Toivonen

Gregg Tolla

G. Tonini

Rebecca Torisky

William F. Tracy

Nicolas Tremblay

Tony Trent

Robert N. Trigiano

Robert R. Tripepi

M.J. Tsujita

P. Tuivonen

Steve Turner

G.A. Tuskan

Hector Valenzuela

Rik van Gorsel

Charles J. Vavrina

Richard E. Veilleux

Peter Vergano

Benigno Villalon

Nicholi Vorsa

Gary W. Wall

Marisa Wall

D.H. Wallace

Robert Wample

W. F. Wardowski

Michele R. Warmund

Debra Warrick

Alley E. Watada

C.B. Watkins

Jon Watterson

Norman F. Weeden

Todd Wehner

Tom C. Weiler

Thomas C. Weiler

Steve Weinbaume

$\mathrm{S}$. Weis
Greg Welbaum

William Welker

Dennis J. Werner

Leslie Weston

Christa Whalen

Raymond Wheeler

Brian Whipker

Anne Whipkey

Bruce D. Whitaker

T.H. Whitlow

Ted Whitwell

Julie Whitworth

Irvin E. Widders

H.C. Wien

Gary Wilfret

Harold Wilkins

Larry Williams

Max W. Williams

Tom Williams

Dale O. Wilson

Farrell C. Wise

Sylvan Wittwer

Tony Wolf

David Wolfe

Dennis J. Wolnick

Bruce Wood

William R. Woodson

Milton Workman

Ray E. Worley

Ron E. Wrolstad

George J. Wulster

Heinz K. Wutscher

Shohjei Yamaki

Ida E. Yates

Thomas Yeager

Keith Yoder

Jayne M. Zajicek

Bernard Zandstra

Francis Zee

Eldon Zehr

Frank Zink

Thomas Zitter

Richard W. Zobel 